\section{RNA POLYMERASE \\ Multiple Forms}

from our Cell Biology Correspondent

BACTERIA, no doubt because of evolutionary pressures, are remarkably economical housekeepers. It is far from easy, for example, to think of ways of improving on nature when the problem is one of providing a unicellular organism with the ability to switch rapidly from the synthesis of one set of protein and RNA molecules to the synthesis of another set at the dictate of the environ. ment. Having basic machinery which has the capacity to catalyse the synthesis of peptide or phosphodiester bonds but lacks the specificity required to decide which particular proteins or RNAs to make until it is programmed by $a$ messenger RNA or a sigma factor is probably the ideal solution. Obviously, it takes far less time and energy to synthesize new programming molecules than to synthesize complete new sets of hardware.

Eukaryotes have during the course of evolution retained this flexible and economic mechanism of protein synthesis and the betting must surely be that they also programme their RNA polymerases with sigma factors. But, equally, it is becoming increasingly clear that in the nuclei of eukaryotic cells there are at least two and possibly, in some if not all cells, three distinct forms of RNA polymerase each of which presumably has some degree of built-in specificity for particular DNA sequences. During the past two years there have been so many reports of two separable RNA polymerases in a range of animal and plant cell nuclei that few cell biologists now question the generality of this finding. Until now, however, three RNA polymerases have only been isolated from one material, developing sea urchins, but in the current Proceedings of the US National Academy of Sciences (68, 338; 1971) Horgen and Griffin report another example of this situation. They have detected three RNA polymerases in extracts of nuclei isolated from the aquatic fungus Blastocladiella emersonii; these three activities can be distinguished not only by their behaviour on chromatography but also with specific inhibitors.

Two of the three polymerase activities resolved by passing extracts of Blastocladiella nuclei through columns of DEAE-cellulose seem homologous to the two activities found in all the eukaryotic nuclei examined to date. One (polymerase II) is sensitive to alpha-amanitin, and like comparable enzymes in other cell-types it no doubt resides in the nucleoplasm where it is probably involved in the synthesis of messenger and other non-ribosomal
RNAs. The second activity (polymerase I), which like its counterparts in other cells is located in the nucleoli (where it probably transcribes ribosomal RNA cistrons), can be specifically inhibited in vitro by cycloheximide, albeit at concentrations far in excess of those required to inhibit protein synthesis. The third polymerase fraction by Horgen and Griffin resembles the RNA polymerase of bacteria in one important respect: it is inhibited by rifampicin. Of course, this enzyme may come from mitochondria which might have contaminated the nuclei isolated from the Blastocladiella cells-it should be possible to test rigorously this suggestion. Alternatively, this third enzyme may be an authentic nuclear polymerase, which, like a vestigial organ, points to the evolutionary ancestry of the fungus.

\section{SKATES \\ Taxonomic Revisions}

from our Marine Vertebrate Correspondent AMONG the cartilaginous fishes the rays and skates have developed a near monopoly of life on the sea floor. Beautifully adapted to exploit a largely two dimensional environment they

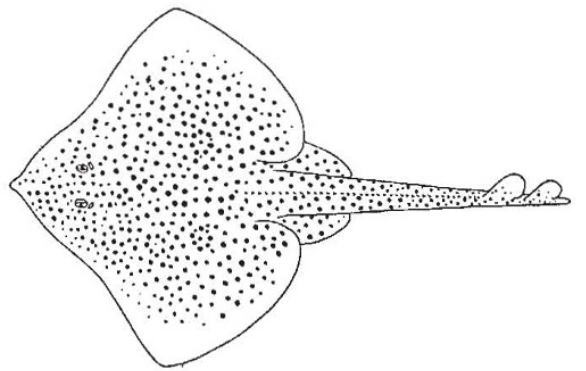

Spotted ray, Raja montagui, found in European waters.

have colonized many tropical fresh waters, the deep sea, and the shallow seas from the Arctic Circle to the Antarctic. With such a wide distribution it is not surprising that very many species have been described and the taxonomy and classification of the group present a very confused situation. Even among the family Rajidae in the north-eastern Atlantic, which includes the moderately important commercial skates, there are many taxonomic problems.

A recent revision of the European skates by M. Stehmann (Arch. Fisch. Wiss., 21, 73; 1970) goes some way to solving some of these problems. Much of the earlier confusion in the

\title{
Explosive Nucleosynthesis in Supernovae
}

SinCE the classic article by Burbidge, Burbidge, Fowler and Hoyle in 1957, it has been generally agreed that the heavy elements were produced by nuclear reactions in stars. Initially, the nuclear processes seemed fairly well understood but their association with particular types of stars was less clear. Recently, work by Arnett and his collaborators has suggested that the relative abundances of essentially all nuclei between neon and nickel can be explained by explosive burning of carbon, oxygen and silicon in supernovae. It is desirable to test these theoretical predictions concerning supernova explosion in two ways. First, are the properties of observed supernovae similar to those predicted by theory and, second, can the total abundances of the moderately heavy element be explained by an acceptable number of supernova explosions?

In next Monday's Nature Physical Science, M. V. Delano reports his studies of the second question. He estimates that the mass of the moderately heavy elements and unburnt lighter elements which must have been expelled by supernovae is of the order of $3.5 \times 10^{9}$ solar masses. The best estimate of the current rate of supernova explosions in galaxies similar to our own is about one every 23 years. If the supernova rate in the galactic life of about $10^{10}$ years has never exceeded that value, an average of 8 solar masses must have been expelled in each explosion. Observations that only the oldest stars are very deficient in heavy elements indicate, however, that a large fraction of these elements were produced early in the galactic history so that the supernova rate was probably much higher then and the mass expelled by each supernova can have been correspondingly less.

Delano has considered a variety of possible past rates of supernovae and values of supernova masses and ejected mass. He concludes that the best agreement between theoretical supernova models and the total production of heavy elements is based on the assumption that supernovae of about 5 solar masses exploded at a rate of about 10 per year for the first few per cent of the galactic lifetime and that there has been a relatively steady and much lower rate since then.

Such a description may be correct, but there are still unsolved problems concerned with the comparison of theoretical and observed supernovae. Very few supernovae have been studied in detail but the estimates of ejected masses are generally lower than the amount required for nucleosynthesis and there is no clear evidence that supernova debris is excessively rich in heavy elements. In addition, some theoretical calculations produce objects which expel masses of process material but never become very luminous. Invisible supernovae may play an important part in nucleosynthesis. Alternatively, explosions in galactic nuclei may be a significant source of heavy elements. 